\title{
Covid-19 Pneumonia with Acute Pulmonary Embolism in a Morbidly Obese Patient; At Home Dramatic Reversal Management
}

\author{
Yasser Mohammed Hassanain Elsayed \\ Critical Care Unit, Kafr El-Bateekh Central Hospital, Damietta Health Affairs, Egyptian Ministry of Health (MOH), Damietta, Egypt.
}

Corresponding Author: Yasser Mohammed Hassanain Elsayed, Critical Care Unit, Kafr El-Bateekh Central Hospital, Damietta Health Affairs, Egyptian Ministry of Health (MOH), Damietta, Egypt.

Received Date: 19 September 2021 | Accepted Date: 28 December 2021 | Published Date: 07 January 2022

Citation: Y M H Elsayed. (2022). Covid-19 Pneumonia with Acute Pulmonary Embolism in a Morbidly Obese Patient; At Home Dramatic Reversal Management. Endocrinology and Disorders. 6(1): DOI:10.31579/2640-1045/113

Copyright: (C) 2022 Yasser Mohammed Hassanain Elsayed, This is an open-access article distributed under the terms of the Creative Commons Attribution License, which permits unrestricted use, distribution, and reproduction in any medium, provided the original author and source are credited.

\begin{abstract}
Rationale: A novel COVID-19 with the severe acute respiratory syndrome had arisen in Wuhan, China in December 2019 Thromboembolism is a critical clinical entity commonly recognized sequel in COVID-19 patients. Interestingly, the presentation of COVID-19 infection with thromboembolism has a risk impact on both morbidity and mortality in COVID-19 patients. Morbid obesity may add over significant risk value in the presence of COVID-19 pneumonia with thromboembolism.

Patient concerns: Middle-aged housewife female COVID-19 morbid obese patient presented to physician outpatient clinic with unilateral pneumonia suspected acute pulmonary embolism.

Diagnosis: COVID-19 pneumonia with acute pulmonary embolism in morbid obesity.

Interventions: CT pulmonary angiography, non- contrasted chest CT scan, electrocardiography, and oxygenation.

Outcomes: Dramatic of both clinical and radiological improvement had happened. Lessons: The combination of morbid obesity, QTc prolongation with COVID-19 infection is an indicator of the over-risk of thromboembolism. It signifies the role of anticoagulants, antiplatelet, anti-infective drugs, and steroids in COVID-19 patients with unilateral pneumonia and acute pulmonary embolism in morbid obesity are effective therapies. An increasing the dose of both low-molecular heparin and oral anticoagulant with a morbidly obese patient was reasonable.
\end{abstract}

Keywords: Covid-19; coronavirus; pneumonia; thromboembolism; pulmonary embolism

\section{Introduction}

The primary presentation of a current Coronavirus-2 (COVID-19) that is causing vigorous acute respiratory syndrome (SARS) had emerged in Wuhan, China in December 2019 [1]. COVID-19 Disease is an extremely communicable, expeditiously spread, deadly worldwide disease [2]. Despite COVID-19 disease was initially appeared with respiratory symptoms, but cardiovascular affection was frequent rather than the presence of higher rates $^{3}$. Indeed, cardiac complications may be manifested as a late phenomenon in respiratory COVID-19 patients. Thromboembolism is one of the most common cardiovascular complications among COVID-19 patients [3]. There is a higher incidence of thromboembolism in patients with SARS-CoV2 disease. These complications are seen in multiple organ failure starting from cutaneous thrombosis to pulmonary embolism, cerebrovascular accident, and coronary thrombosis [4]. In some observational studies, thrombotic complications were reported as high as $31 \%$ in patients indicating critical care admission [4]. The essential role of thrombo-inflammation and endothelial injury in the pathogenesis of the COVID-19 is clearly detected. Overproduction of pro-inflammatory cytokines such as tumor necrosis factor (TNF), Interleukin (IL)-6, IL-8, and IL-1 $\beta$ that is known as "cytokine release syndrome" or "cytokine storm" not unique to this disease. Also, it had described in sepsis and sterile inflammation [4]. This exaggerated cytokine response may lead to multiorgan failure and eventually death in some patients [5]. A high index of clinical suspicion 
for thrombotic status and their consequences is needed for accurate and quick diagnosis [4]. The optimal approach to the management of anticoagulation in these patients is still obscure in absence of wellconducted trials. There remains major uncertainty in the optimal management of immune-thrombosis as commonly seen in COVID-19 [4]. Both unfractionated heparin and low molecular weight heparin (LMWH) succeed in these patients both prophylactic and therapeutic measures [6, 7]. Higher doses should be considered for those with higher risk patients (eg, obese, active malignancy, prolonged immobility, or recent surgery) [4]. Obesity is associated with numerous pathological conditions, including venous thromboembolism (VTE). Obesity is an independent and moderate risk factor for VTE. The risk increases with body mass index, age, and other acquired risk factors [8].

\section{Case presentation}

A 45-year-old married housewife Egyptian female patient presented to the physician outpatient clinic (POC) with acute tachypnea and dizziness. Generalized malaise, loss of appetite, loss of smell and taste, and generalized body aches were associated symptoms. The patient gave a history of the symptoms for 6 days. Currently, she had a history of contact with her friend who confirmed a COVID-19 patient in the past 14 days. Informed consent was taken. Upon general physical examination; generally, the patient was tachypneic, distressed, with an irregular pulse rate (sinus arrhythmia with VR; $80 \mathrm{bpm}$ ), blood pressure (BP) of 130/70 $\mathrm{mmHg}$, respiratory rate of $32 \mathrm{bpm}$, the temperature of $37.3^{\circ} \mathrm{C}$, and pulse oximeter of oxygen $(\mathrm{O} 2)$ saturation of $91 \%$. She appeared very obese with a lasting body weight (one week ago) of $238 \mathrm{~kg}$ and a height of $164 \mathrm{~cm}$. According to these weight lengths; calculated BMR was very high (78.6). No more relevant clinical data were noted during the clinical examination. The patient was treated at home with COVID-19 pneumonia with suspected acute pulmonary embolism. Initially, the patient was treated with $\mathrm{O} 2$ inhalation by $\mathrm{O} 2$ cylinder $(100 \%$, by nasal cannula, $5 \mathrm{~L} / \mathrm{min})$. The patient was maintain treated with cefotaxime; (1000 mg IV every 8hours), azithromycin (500 mg PO single daily dose), oseltamivir (75 mg PO twice daily only for 5 days), and paracetamol (500 mg IV every 8 hours as needed). SC enoxaparin $80 \mathrm{mg}$ twice daily), aspirin tablet $(75 \mathrm{mg}$, once daily), clopidogrel tablet (75 mg, once daily), and hydrocortisone sodium succinate (100 mg IV every 12 hours) were added. Warfarin $5 \mathrm{mg}$ oral tablet was added and adjusted with serial INR and PT. The patient was daily monitored for temperature, pulse, blood pressure, and $\mathrm{O} 2$ saturation. The initial ECG on presentation showing right bundle branch block (RBBB) with sinus arrhythmia of VR of 78 with prolonged QTc-interval (484 ms in lead II). (Figure 1). The initial complete blood count (CBC); $\mathrm{Hb}$ was $10.6 \mathrm{~g} / \mathrm{dl}$, RBCs; $4.03 * 10^{3} / \mathrm{mm}^{3}$, WBCs; $16.6 * 10^{3} / \mathrm{mm}^{3}$ (Neutrophils; $89.1 \%$, Lymphocytes: 7.9\%, Monocytes; 2\%, Eosinophils; $1 \%$ and Basophils 0\%), Platelets; $158 * 10^{3} / \mathrm{mm}^{3}$. S. Ferritin was high; 635 $\mathrm{ng} / \mathrm{ml}$. D-dimer was very high $(2694 \mathrm{ng} / \mathrm{ml})$. CRP was high $(98 \mathrm{~g} / \mathrm{dl})$. LDH was high (668 U/L). SGPT was normal (39 U/L), SGOT was normal (27 U/L). Serum creatinine was normal $(0.7 \mathrm{mg} / \mathrm{dl})$ and blood urea was normal $(25 \mathrm{mg} / \mathrm{dl})$. RBS was normal $(188 \mathrm{mg} / \mathrm{dl})$. Ionized calcium was slightly low $(1.01 \mathrm{mmol} / \mathrm{L})$. The troponin test was negative. After 17 days of management; RBS was normal $(107 \mathrm{mg} / \mathrm{dl})$. CBC; Hb was $11.2 \mathrm{~g} / \mathrm{dl}$, RBCs; $4.10^{*} 10^{3} / \mathrm{mm}^{3}$, WBCs; $8.3 * 10^{3} / \mathrm{mm}^{3}$ (Neutrophils; $66.4 \%$, Lymphocytes: $23.5 \%$, Monocytes; $7.1 \%$, Eosinophils; $3 \%$ and Basophils $0 \%)$, Platelets; $134 * 10^{3} / \mathrm{mm}^{3}$. Serum ferritin was normal $(261 \mathrm{ng} / \mathrm{ml})$. Ddimer was normal $(174 \mathrm{ng} / \mathrm{ml})$. CRP was negative $(0.5 \mathrm{~g} / \mathrm{dl})$. LDH was still high (553U/L). SGPT was normal (22 U/L), SGOT was normal (28 $\mathrm{U} / \mathrm{L})$. Serum creatinine $(1.2 \mathrm{mg} / \mathrm{dl})$ and blood urea $(39 \mathrm{mg} / \mathrm{dl})$ were normal. Ionized calcium was normal; $1.17 \mathrm{mmol} / \mathrm{L}$. The troponin test had still negative. The first CT pulmonary angiography (CTPA) was done on the day of the presentation showing thromboses of the second, distal divisions of the right pulmonary artery, and incomplete filling defects in the superior vena cava. There is single patchy ground-glass pulmonary consolidation in the upper lobe of the right lung. Also, there is right lower lung lobe sub-segmental consolidation (Figure 2A-D). Chest CT without contrast was done within 17 days of treatment showing complete resolution of the above abnormalities (Figure 3). COVID-19 pneumonia with suspected acute pulmonary embolism in morbid obesity was the most probable diagnosis. Within 15 days of the above management, the patient finally showed nearly complete clinical, radiological, and laboratory improvement. The patient was continued on aspirin tablet (75 $\mathrm{mg}$, once daily) and warfarin $8 \mathrm{mg}$ oral tablet with follow-up with serial INR and PT. Further recommended cardiac chest follow-up, and weight reduction was advised.

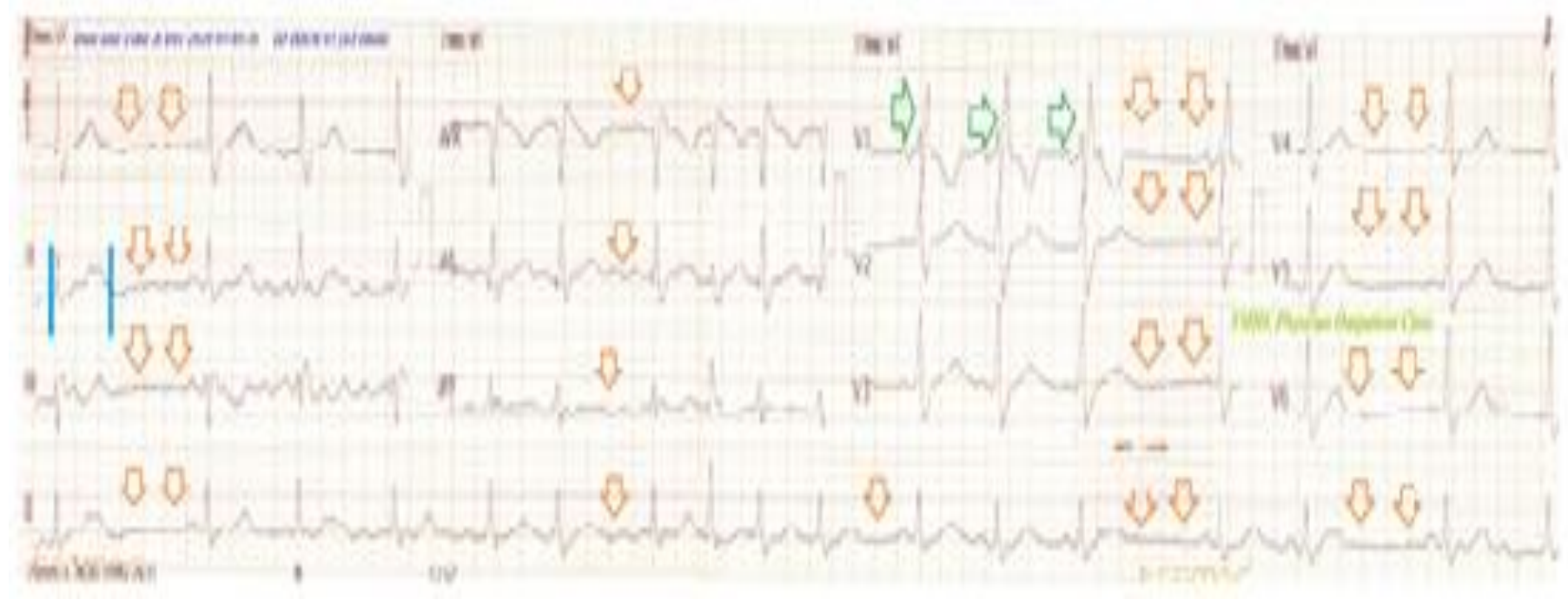

Figure 1: ECG tracings; A. tracing was done on the initial ECG on presentation showing RBBB (green arrows) with sinus arrhythmia (orange arrows) of VR of 78 with prolonged QTc-interval (484 ms; blue vertical lines in lead II). 


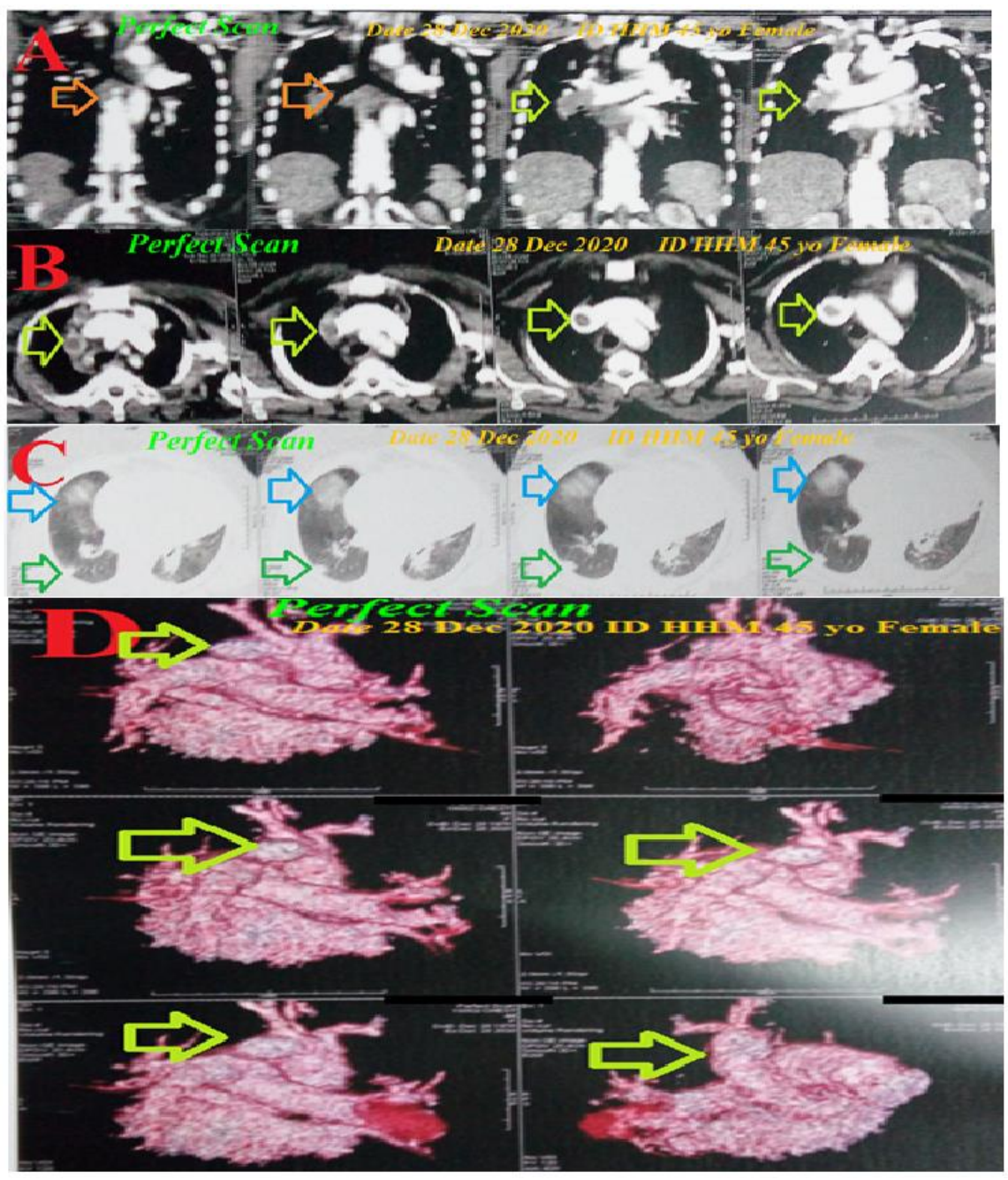

Figure 2: Variable sections of CT pulmonary angiography (CTPA; A-D) was done on the day of the presentation showing thromboses of the second, distal divisions of the right pulmonary artery (lime arrows), and incomplete filling defects in superior vena cava (orange arrows). There is single patchy ground-glass pulmonary consolidation in the upper lobe of the right lung (blue arrows). Also, there is right lower lung lobe sub-segmental consolidation (green arrows).

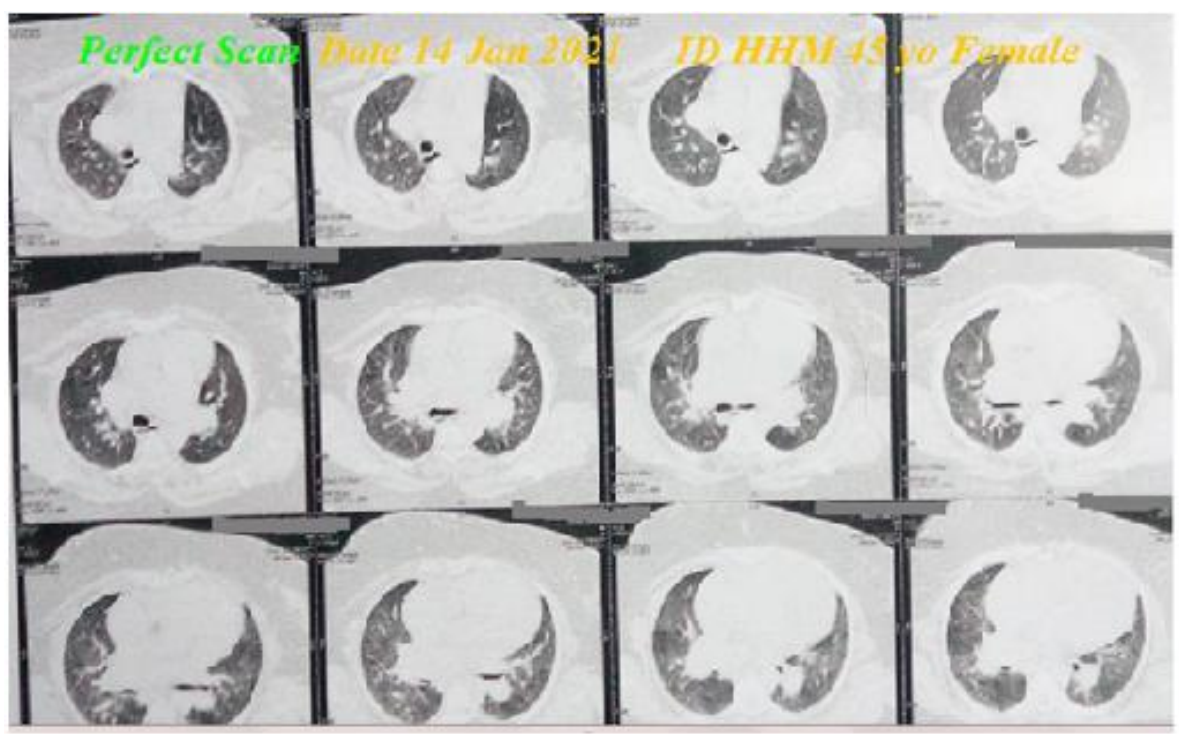

Figure 3: Chest CT without contrast was done within 17 days of treatment showing complete resolution of the above abnormalities. 


\section{Discussion}

- Overview:

- Middle-aged housewife female COVID-19 morbid obese patient presented to physician outpatient clinic with unilateral pneumonia with suspected acute pulmonary embolism.

- The primary objective for my case study was the presence of COVID-19 pneumonia with suspected acute pulmonary embolism in a morbidly obese patient in POC.

- The secondary objective for my case study was the question of; how did you manage the case at home?

- There was a history of direct contact to confirmed the COVID19 case.

- The presence of direct contact to confirmed the COVID-19 case, and unilateral ground-glass consolidation on top of acute tachypnea will strengthen the COVID-19 diagnosis.

- The tachypnea, hypoxia, consolidation, electrocardiographic (ECG) RBBB and elevated d-dimer are highly suggestive of associated pulmonary embolism.

- An associated morbid obesity, ECG RBBB with QTc prolongation, and marked elevated d-dimer in the presence of COVID-19 pneumonia may carry a bad prognostic outcome and is indicating a high-risk condition.

- An accompanied ECG sinus arrhythmia is insignificant and valueless.

- An increasing the dose of both low-molecular heparin (enoxaparin) and oral anticoagulant (warfarin) with a morbidly obese patient was reasonable.

- The dramatic reversal of clinical, electrocardiographic, and radiological after anticoagulants, antiplatelet, anti-infective drugs, and steroids had happened.

- A nearly complete clinical, radiological, and laboratory improvement that occurred after the management with antiinfective drugs, anticoagulants, steroids, and antiplatelet strongly implies their effects.

- Blood pressure, respiratory rate, pulse, and $\mathrm{O} 2$ saturation are a strong guide for clinical follow-up in COVID-19 patients.

- A gradual decreasing the level of elevated CRP, d-dimer, and serum ferritin may be used as another good laboratory guide for follow-up for COVID-19 pneumonic patients.

- Acute coronary syndrome was the possible differential diagnosis for the current case study.

- I can't compare the current case with similar conditions. There are no similar or known cases with the same management for near comparison.

- The only limitation of the current study was the unavailability of echocardiography.

\section{Conclusion and Recommendations}

The combination of morbid obesity, QTc prolongation with COVID-19 infection is an indicator of the over-risk of thromboembolism. It signifies the role of anticoagulants, antiplatelet, anti-infective drugs, and steroids in COVID-19 patients with unilateral pneumonia and acute pulmonary embolism in morbid obesity are effective therapies.

An increasing the dose of both low-molecular heparin and oral anticoagulant with a morbidly obese patient was reasonable.

\section{Abbreviations}

CBC: Complete blood count

COVID-19: Coronavirus disease 2019

CTPA: CT pulmonary angiography

ECG: Electrocardiography

IV: Intravenous

O2: Oxygen

POC: Physician outpatient clinic

RBBB; Right bundle branch block

SGOT: Serum glutamic-oxaloacetic transaminase

$S G P T$ : Serum glutamic-pyruvic transaminase

VR: Ventricular rate

VTE: Venous thromboembolism

\section{Conflicts of interest}

There are no conflicts of interest.

\section{Acknowledgment}

I wish to thanks the nurse team of the intensive care unit to give me extra copies of the ECG to help me and my wife to save time and improving the conditions for supporting me.

\section{References}

1. Zhu N, Zhang D, Wang W, et al. (2020). A novel coronavirus from patients with pneumonia in China, 2019. N Engl J Med. 382:727-733.

2. WHO. Coronavirus disease 2019 (COVID-19) Situation Report -165 .

3. Kwenandar F, Japar KV, Damay V, Hariyanto TI, Tanaka M, Lugito NPH, Kurniawan A. (2020). Coronavirus disease 2019 and cardiovascular system: A narrative review. Int J Cardiol Heart Vasc. 29:100557.

4. Mondal S, Quintili A L, Karamchandani K. et al. (2020). Thromboembolic disease in COVID-19 patients: A brief narrative review. $\mathrm{j}$ intensive care. 8:70.

5. Meduri GU, Kohler G, Headley S, Tolley E, Stentz F, Postlethwaite A. (1995). Inflammatory cytokines in the BAL of patients with ARDS. Persistent elevation over time predicts poor outcome. Chest. 108(5):1303-1314.

6. Thachil J, Tang N, Gando S, et al. (2020). Type and dose of heparin in COVID-19. J Thromb Haemost. 18(8):2063-2064.

7. Belen-Apak FB, Sarialioglu F. (2020). The old but new: Can unfractioned heparin and low molecular weight heparins inhibit proteolytic activation and cellular internalization of SARSCoV2 by inhibition of host cell proteases? Med Hypotheses. 142:109743.

8. Hotoleanu C. (2020). Association between obesity and venous thromboembolism. Med Pharm Rep. 93(2):162-168. 